\title{
Modification of Cellulose from water hyacinth (Eichhornia crassipes) for nanocomposite materials
}

\author{
Hien V. Nguyen ${ }^{1 *}$, Thuy T. T. Le ${ }^{2}$, \& Duy Q. Tran ${ }^{2}$ \\ ${ }^{1}$ Faculty of Science, Nong Lam University, Ho Chi Minh City, Vietnam \\ ${ }^{2}$ Department of Chemical Engineering, Nong Lam University, Ho Chi Minh City, Vietnam
}

\begin{abstract}
ARTICLE INFO
Research Paper

Received: November 23, 2018

Revised: April 10, 2019

Accepted: April 22, 2019

\section{Keywords}

Cellulose acetate

Nanocomposite materials

Water hyacinth

\section{*Corresponding author}

Nguyen Van Hien

Email: nvhien@hcmuaf.edu.vn
\end{abstract}

\section{ABSTRACT}

The effects of temperature, time, alkalinity and solid/liquid ratio on separation of lignin, hemicellulose to obtain cellulose from water hyacinth (Eichhornia crassipes) were investigated. Microcrystalline cellulose fibers were examined by microscope and infrared spectroscopy IR. The results showed that lignin and hemicellulose were removed by alkaline treatment. The obtained cellulose fibers were modified by esterification reaction with acetic anhydride in acetic acid, sulfuric acid with the aid of ultrasound. The cellulose acetate was investigated using transmission electron microscopy (TEM) and IR spectra. The synthesized nano fibers were in the diameter range of $50-100 \mathrm{~nm}$ from the TEM with even arrangement. The IR spectrum also showed that the $-\mathrm{OH}$ group was replaced by the $-\mathrm{OCOCH}_{3}$ group, thus, reducing the polarization and hygroscopic ability of cellulose, suitable for using as the reinforcing phase in biodegradable composite materials.

Cited as: Nguyen, H. V., Le, T. T. T., \& Tran, D. Q. (2019). Modification of Cellulose from water hyacinth (Eichhornia crassipes) for nanocomposite materials. The Journal of Agriculture and Development 18(4), 26-32. 


\title{
Nghiên cứu biến tính Cellulose từ lục bình định hướng làm pha gia cường cho vật liệu nanocomposite
}

\author{
Nguyễn Văn Hiền ${ }^{1 *}$, Lê Thị Thanh Thủy ${ }^{2}$ \& Trần Quang Duy ${ }^{2}$ \\ Bộ Môn Công Nghệ Hóa Học, Trường Đại Học Nông Lâm TP.HCM, TP. Hồ Chí Minh
}

THÔNG TIN BÀI BÁO
Bài báo khoa học
Ngày nhận: 23/11/2018
Ngày chînh sửa: 10/04/2019
Ngày chấp nhận: 22/04/2019
Từ khóa
Cellulose acetate
Lục bình
Vật liệu nanocomposite
*Tác giả liên hệ
Nguyễn Văn Hiền
Email: nvhien@hcmuaf.edu.vn

\section{TÓM TẮT}

Quá trình tách lignin, hemicellulose để thu được cellulose từ lục bình (Eichhornia crassipes) bằng phương pháp xử lý kiềm dưới ảnh hưởng của các yếu tố: nhiệt độ, thời gian, nồng độ kiềm và tỉ lệ rắn/lỏng. Vi sợi cellulose được khảo sát cấu trúc bằng kính hiển vi và phổ hồng ngoại IR. Kết quả thu được cho thấy chỉ cần qua một bước xử lý kiềm đã hoàn toàn loại bỏ được lignin và hemicellulose. Sợi cellulose tiếp tục được thử nghiệm biến tính bằng phản ứng ester hóa với tác nhân anhydride acetic trong dung môi acid acetic, xúc tác H2SO4 đặc, có sự hỗ trợ của sóng siêu âm. Sản phẩm cellulose acetate được đánh giá kích thước, hình thái và tính chất bằng kính hiển vi điện tử truyền qua (TEM), phổ hồng ngoại (IR). Kết quả cho thấy vi sợi celullose acetate có kích thước khoảng $50 \mathrm{~nm}$, phân bố khá đồng đều. Đồng thời trên phổ IR cho thấy nhóm $-\mathrm{OH}$ đã được thay thế bằng nhóm $-\mathrm{OCOCH}_{3}$, từ đó làm giảm độ phân cực và tính hút ẩm của cellulose, phù hợp định hướng làm pha gia cường cho vật liệu composite phân hủy sinh học.

\section{1. Đặt Vấn Đề}

Sự phát triển mạnh mẽ của công nghiệp hiện đại dẫn đến nhu cầu ngày càng lớn về việc nghiên cứu, chế tạo các loại vật liệu mới. Vật liệu nanocomposite là sự kết hợp của hai hay nhiều vật liệu khác nhau, mỗi thành phần vẫn giữ được những tính chất cơ, lý, hóa riêng biệt của nó. Đồng thời vật liệu nanocomposite có những tính chất tốt hơn so với từng vật liệu ban đầu. Gần đây, các nghiên cứu mới nỗ lực phát triển vật liệu nanocomposite từ nguồn gốc tự nhiên có khả năng phân hủy sinh học, nhằm giảm thiểu tối đa tác hại đối với môi trường (Oka, 2010). Sợi nano cellulose có tiềm năng lớn để làm pha gia cường cho các vật liệu composite sinh học như khả năng phân tán cao, đồng đều, không độc và có khả năng phân hủy sinh học. Tuy nhiên trong cấu trúc của cellulose có nhiều nhóm - $\mathrm{OH}$ nên có tính phân cực, do đó để tăng độ phân tán và tương thích trong nền polymer cần biến tính các nhóm - $\mathrm{OH}$, để làm giảm tính phân cực của cellulose (Wang \& Lawrence, 2012). Acetyl hóa là một phương pháp đầy hứa hẹn, trong đó một số nhóm -OH phản ứng với tác nhân anhydride acetic để tạo thành sản phẩm cellulose acetate $(\mathrm{CA})$ có độ phân cực kém hơn cellulose, giúp giảm tính hút ẩm của cellulose và phân tán tốt hơn trong trong nền polymer (Abdulkhani \& ctv., 2014).

Lục bình (Eichhornia crassipe) là một loài thực vật thủy sinh sống trôi nổi, hay cắm rễ xuống bùn, rễ chùm, sinh trưởng rất nhanh, khó kiểm soát, ảnh hưởng không chỉ đến tính đa dạng sinh học mà còn ảnh hưởng đến các hoạt động kinh tế - xã hội. Xuất phát từ vùng Amazon, Nam Mỹ, đến nay lục bình đã phát triển trên 50 quốc gia vùng nhiệt đới và cận nhiệt đới (Abdulkhani \& ctv., 2014). Ở Việt Nam, trong những năm qua, sự xuất hiện của lục bình trên các kênh rạch thuộc các khu vực sông ngày càng nhiều và dày đặc. Cụ thể tại thành phố Hồ Chí Minh, đã có khoảng 200 km thuộc 180 kênh rạch bị xâm chiếm bởi lục bình. Nó không những cản trở dòng chảy, gây khó khăn cho giao thông đường thủy, lục bình còn gây ô nhiễm, ảnh hưởng đến nguồn nước phục vụ tưới tiêu đồng ruộng cũng như nuôi trồng thủy 
sản tại một số nơi. Hiện nay có nhiều giải phát được đưa ra để giải quyết sự ô nhiễm của lục bình, nhưng hiệu quả vẫn còn chưa cao. Do lục bình có hàm lượng cellulose cao (trên $200 \mathrm{~g} / 1 \mathrm{~kg}$ ) nên cũng có thể tận dụng nguồn cellulose từ loại thực vật này (Istirokhatun \& ctv., 2015). Vì vậy, mục tiêu của nghiên cứu này là tách lấy cellulose từ lục bình, biến tính tạo cellulose acetate định hướng làm pha gia cường trong tổng hợp vật liệu nanocomposite phân hủy sinh học.

\section{Vật Liệu và Phương Pháp Nghiên Cứu}

\subsection{Vật liệu}

Lục bình được thu gom từ ao, hồ khu vực quận Thủ Đức, TP.HCM, với kích thước thân cây trên 0,5 mét.

Toluene, Etanol 96\%, Acid acetic, Natri hydroxid 96\%, Acid chlorhidric 36\%, Sodium hypochlorite $(\mathrm{NaClO})$ có nguồn gốc Trung Quốc và Anhydride acetic từ Tây Ban Nha.

\subsection{Phương pháp}

Xử lý lignin và hemicellulose từ lục bình: Lục bình tươi được rửa sạch, cắt nhỏ, nghiền và sấy khô, sau đó ngâm dầm trong dung môi Toluene/Ethanol trong 24 giờ, nhằm loại bỏ các chất nhựa và các chất mang màu. Sau bước xử lý bằng dung môi, mẫu lục bình được cho vào bình phản ứng chứa dung dịch $\mathrm{NaOH}$ (nồng độ và tỉ lệ sẽ được khảo sát), đun hồi lưu với nhiệt độ và thời gian thích hợp. Sau đó rửa lại bằng dung dịch $\mathrm{HCl} 0,5 \mathrm{M}$ rồi tách dịch lọc, bã được rửa bằng nước cất rồi đem đi sấy khô. Mẫu khô được tiếp tục được tẩy lignin bằng phản ứng với dung dịch $\mathrm{NaClO}$. Hỗn hợp sau phản ứng được rửa nhiều lần bằng nước cất, sau đó tách dịch lọc. Bã sau khi lọc được sấy khô ở $60^{\circ} \mathrm{C}$ và cân lại khối lượng để tính hiệu suất của các phản ứng trên. Cellulose thu được sau khi sấy khô được kiểm tra tính chất bằng phổ hồng ngoại IR (Trung tâm INOMAR, Đại học Quốc gia TP.HCM).

Tổng hợp cellulose acetate: Cần chính xác 0,5 $\mathrm{g}$ bột cellulose cho vào bình cầu $50 \mathrm{~mL}$, thêm $10 \mathrm{~mL}$ acid acetic và siêu âm 10 phút để cellulose phân tán đều trong dung môi. Tiếp tục thêm anhydride acetic $(5,7,10,13 \mathrm{~mL})$ và acid $\mathrm{H}_{2} \mathrm{SO}_{4}$ đặc vào hỗn hợp phản ứng, siêu âm trong 30 phút -3 giờ. Sau thời gian phản ứng, tiến hành kết tủa và thu vi sợi cellulose acetate. Sản phẩm được kiểm tra tính chất bằng phương pháp kính hiển vi điện tử truyền qua TEM (Phòng thí nghiệm Trọng điểm Polymer và Composite, Trường Đại học Bách Khoa TP.HCM) và phổ hồng ngoại IR (Trung tâm INOMAR, Đại học Quốc gia TP.HCM).

\section{Kết Quả và Thảo Luận}

\subsection{Tách cellulose từ lục bình}

\subsubsection{Kết quả tách cellulose từ lục bình}

Quá trình tách sợi cellulose từ lục bình khô được thực hiện qua 2 bước: xử lý hemicellulose (0,5 g lục bình khô trong dung dịch $\mathrm{NaOH} 0,25$ $\mathrm{M}$, phản ứng trong 3 giờ $)$ và xử lý lignin $(0,2 \mathrm{~g}$ mẫu sau bước 1 với dung dịch $\mathrm{NaClO} 2 \%$, phản ứng trong 2 giờ). Tỷ lệ phần trăm hao hụt về khối lượng sau các quá trình xử lý được thể hiện trong Bảng 1.

Bảng 1. Hiệu suất sau các quá trình xử lý

\begin{tabular}{lc}
\hline Bước xử lý & $\begin{array}{c}\text { Tỷ lệ } \\
\text { hao } \\
\text { hụt }\end{array}$ \\
\hline $\begin{array}{l}\text { 1. Xử lý dung môi (Toluene/Ethanol) } \\
\text { 2. Xử lý hemicellulose 38,5\% }\end{array}$ & $10,2 \%$ \\
3. Xử lý lignin & $3,7 \%$ \\
Tồng & $52,4 \%$ \\
\hline
\end{tabular}

Như vậy, sau quá trình xử lý dung môi, xử lý hemicelluloses và lignin, tổng khối lượng các chất được loại bỏ bằng 52,4\% so với tổng khối lượng lục bình khô ban đầu.

Mẫu cellulose thu được sau khi sây khô ở $60^{\circ} \mathrm{C}$ được phân tích phổ hồng ngoại IR, kết quả được thể hiện trong Hình 1.

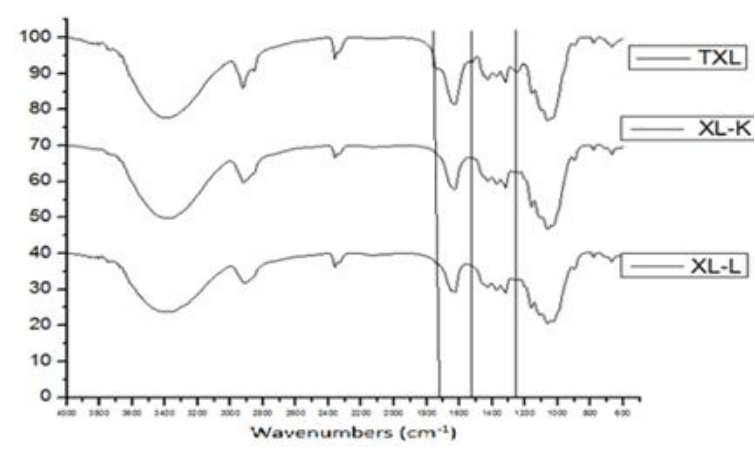

Hình 1. Giản đồ phổ IR qua các bước xử lý mẫu. (TXL: Tiền xử lý, XL-K: Xử lý kiềm, XL-L: Xử lý lignin). 
Kết quả phân tích hồng ngoại cho phép đánh giá sự có mặt của các nhóm chức cũng như khẳng định phần nào cấu trúc phân tử của chúng. Giản đồ phổ IR cho thấy so với mẫu tiền xử lý, các mẫu đã xử lý hemicellulose và lignin có vài điểm khác biệt (chứng tỏ quá trình xử lý đạt hiệu quả): peak nhỏ ở số sóng 1734/cm đặc trưng cho dao động nhóm cacbonyl trong các nhóm acetyl và este đã biến mất trong các mẫu đã xử lý, 2 nhóm chức này đặc trưng cho sự có mặt của hemicellulose và lignin. Peak ở số sóng $1519 / \mathrm{cm}$ và peak ở số sóng $1253 / \mathrm{cm}$ đặc trưng cho dao động liên kết C-C trong vòng thơm (chỉ có ở cấu trúc lignin) cũng biến mất trong 2 mẫu đã xử lý (Nguyen \& ctv., 2017). Các kết quả trên cho thấy quá trình xử lý đã đạt được hiệu quả như mong muốn, các thành phần như hemicelllulose và lignin (đặc biệt là lignin) đã bị loại bỏ, cấu trúc cellulose được giữ lại (thể hiện qua các peak ở số sóng 2800 $2900 / \mathrm{cm}$ và $3300-3500 / \mathrm{cm}$ đặc trưng cho liên kết $\mathrm{C}-\mathrm{H}$ và $\mathrm{O}-\mathrm{H}$ trong cấu trúc cellulose được giữ nguyên) (Nguyen \& ctv., 2017).

Ngoài ra, khi so sánh phổ IR của 2 quá trình xử lý hemicellulose và lignin sẽ không thấy sự khác biệt. Điều này có thể cho thấy dung dịch kiềm có thể cho hiệu quả ở cả 2 quá trình xử lý hemicellulose và lignin. Từ đó nhóm nghiên cứu đề xuất chỉ sử dụng dung dịch kiềm cho cả 2 quá trình xử lý (xử lý 1 bước).

\subsubsection{Khảo sát ảnh hưởng nồng độ $\mathrm{NaOH}$ đến quá trình xử lý hemicellulose và lignin}

Quá trình xử lý kiềm làm phá vỡ thành tế bào và liên kết $\alpha$-ete, ester giữa lignin, hemicellulose, và các acid khác. Do đó nồng độ $\mathrm{NaOH}$ là một trong những yếu tố quan trọng ảnh hưởng đến hiệu suất quá trình xử lý.

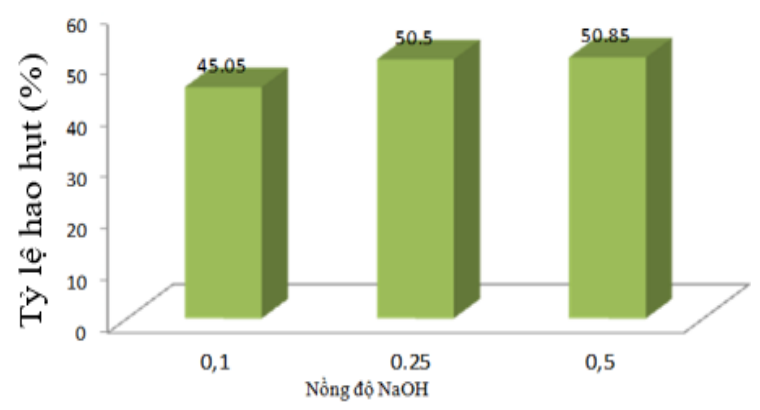

Hình 2. Ảnh hưởng của nồng độ $\mathrm{NaOH}$ đến phần trăm hao hụt khối lượng sau khi xử lý hemicellulose và lignin từ lục bình.
Dựa vào biểu đồ Hình 2 thấy được khi tăng nồng độ $\mathrm{NaOH}$ từ 0,1 lên $0,25 \mathrm{M}$ thì tỷ lệ hao hụt khối lượng tăng lên đáng kể, từ 45,05\% lên $50,5 \%$. Tuy nhiên khi tăng nồng độ $\mathrm{NaOH}$ lên 0,5 $\mathrm{M}$ thì hiệu suất gần như không đổi. Như vậy hàm lượng hemicellulose và lignin trong lục bình chỉ bị hòa tan trong một nồng độ kiềm phù hợp nhất định. Để tiết kiệm năng lượng và chi phí dựa vào biểu đồ ta chọn nồng độ dung dịch kiềm $\mathrm{NaOH}$ $0,25 \mathrm{M}$ làm điều kiện thích hợp cho quá trình xử lý hemicellulose và lignin.

\subsubsection{Khảo sát ảnh hưởng của thời gian đến quá trình xử lý hemicellulose và lignin}

Từ biểu đồ Hình 3 thấy được thời gian có ảnh hưởng đến quá trình xử lý hemicellulose và lignin. Tỷ lệ khối lượng bị hao hụt sau quá trình xử lý có xu hướng tăng cao khi tăng thời gian từ 2 giờ $(25,9 \%)$ đến 4 giờ $(50,5 \%)$. Tuy nhiên khi tăng thời gian từ 4 giờ đến 6 giờ thì tỷ lệ này tỷ lệ này thay đổi không đáng kể, cụ thể lần lượt là $50,5 \%, 51,65 \%$ và $51,85 \%$. Từ kết quả trên cho thấy thời gian 5 giờ là phù hợp cho quá trình xử lý hemicellulose và lignin tại nồng độ $\mathrm{NaOH}$ là $0,25 \mathrm{M}$.

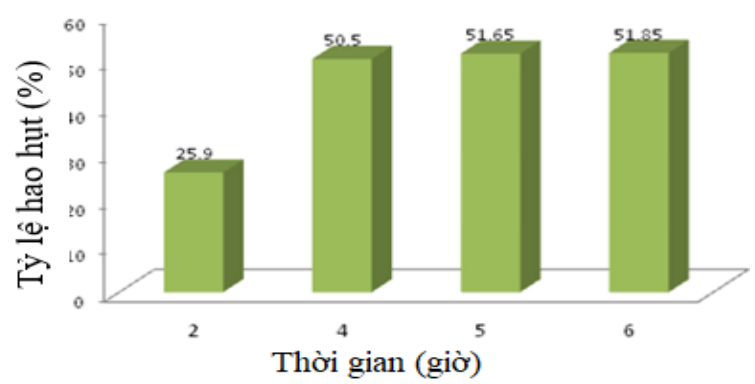

Hình 3. Ảnh hưởng của thời gian đến tỷ lệ hao hụt khối lượng sau khi xử lý hemicellulose và lignin từ lục bình.

Như vậy, điều kiện phù hợp cho quá trình xử lý hemicellulose và lignin trong lục bình là: $0,5 \mathrm{~g}$ lục bình khô trong $25 \mathrm{~mL}$ dung dịch $\mathrm{NaOH} \mathrm{0,25}$ $\mathrm{M}$, thời gian 5 giờ.

\subsection{Biến tính cellulose bằng phản ứng ester hóa}

3.2.1. Kết quả biến tính cellulose bằng phản ứng ester hóa

Cellulose thu được sau quá trình xử lý hemicellulose và lignin từ lục bình, được tiến hành phản 
ứng ester hóa bằng anhydride acetic trong dung môi acid acetic và xúc tác acid sulfuric đặc. Sản phẩm được kiểm tra bằng phương pháp IR. Kết quả phân tích được thể hiện như Hình 4.

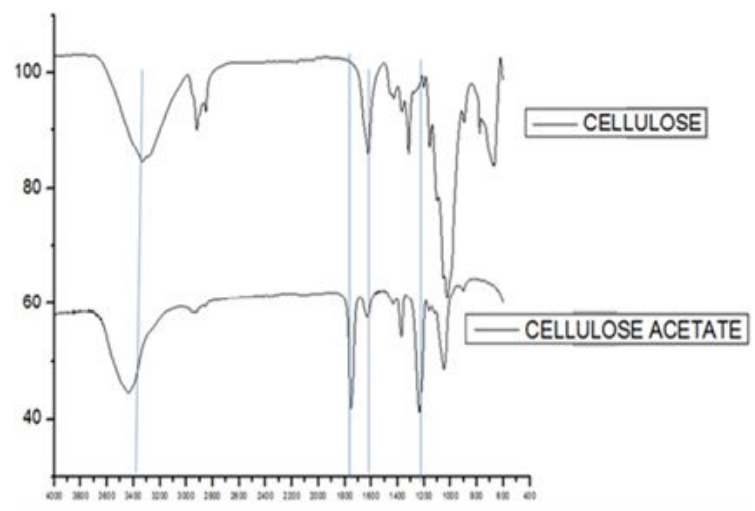

Hình 4. Giản đồ phổ IR trước và sau khi acetate hóa.

Qua hai giản đồ IR của cellulose và cellulose acetate ta có nhận thây: peak O-H ở số sóng 3300 - 3500/cm cường độ giảm, chứng tỏ một phần nhóm - $\mathrm{OH}$ đã bị acetate hóa. Ngoài ra peak này có hình dạng nhọn hơn là do cường độ liên kết hydro giữa các nhóm - $\mathrm{OH}$ giảm, cũng cho thấy rằng một số nhóm - $\mathrm{OH}$ trong cấu trúc cellulose đã bị thay thế. So với cellulose, trên giản đồ IR của cellulose acetate xuất hiện 2 peak mới ở 1730 và $1247 / \mathrm{cm}$ tương ứng với dao động của các liên kết $\mathrm{C}=\mathrm{O}$ và $\mathrm{C}-\mathrm{O}$ trong nhóm ester, chứng tỏ quá trình acetate hóa đạt hiệu quả. Ngoài ra, peak ở $1600-1650 / \mathrm{cm}$ thường đặc trưng cho việc hấp thụ hơi nước của các chất, peak này ở mẫu cellulose acetate có cường độ giảm đáng kể so với mẫu cellulose, cho thấy sản phẩm sau khi acetate hóa có độ phân cực giảm. Điều này có thể được giải thích là do quá trình acetate hóa cellulose, một số nhóm - $\mathrm{OH}$ được thay bằng các nhóm $-\mathrm{OCOCH}_{3}$ làm giảm độ phân cực, việc hấp thụ nước cũng giảm so với cellulose. Việc làm giảm độ phân cực của cellulose đạt được mục đích đề ra ban đầu trong việc định hướng làm pha gia cường trong tổng hợp composite (vì pha gia cường kém phân cực mới tương thích với các nền polymer kém phân cực). Mặt khác sự vắng mặt của các peak trong phạm vi số sóng từ 1760 đến 1840/cm chứng tỏ anhydride acetic và acid acetic đã được loại bỏ hoàn toàn.

Mẫu cellulose acetate thu được sau khi acetate hóa trắng và có độ mịn cao (Hình 5). Có thể dự đoán trong quá trình acetate hóa đồng thời đã làm giảm kích thước sợi cellulose. Điều này sẽ được kiểm tra bằng phương pháp kính hiển vi (Hình 6) và kính hiển vi điện tử truyền qua TEM (Hình 7) để đánh giá kích thước sợi cellulose acetate.

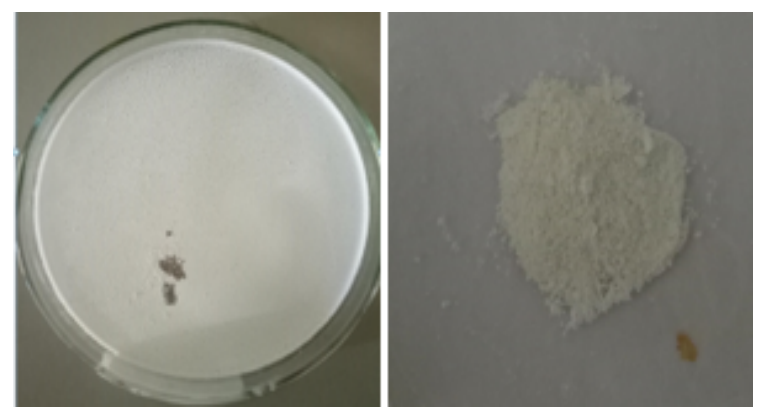

Hình 5. Mẫu cellulose sau khi acetate hóa.

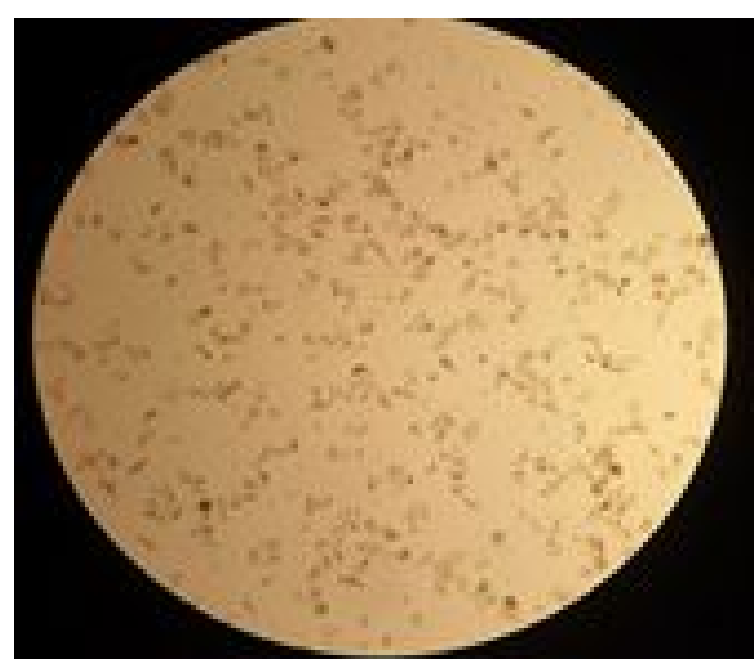

Hình 6. Mẫu được chụp qua kính hiển vi sau khi acetate hóa (độ phóng đại 1000).

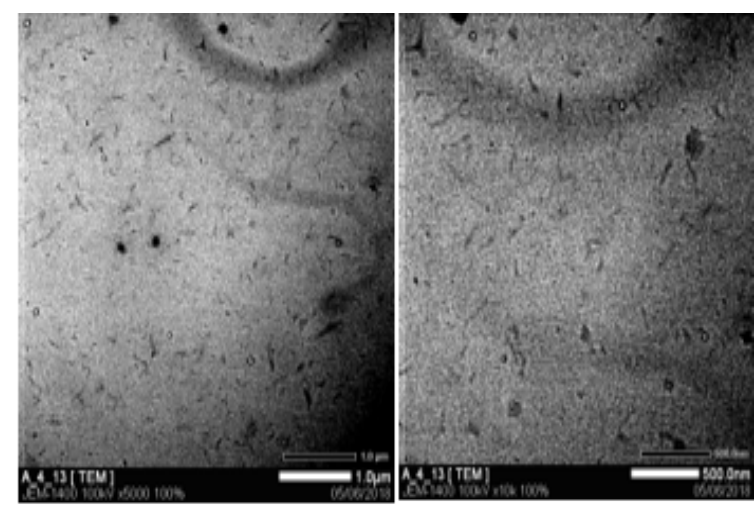

Hình 7. Mẫu được chụp bằng phương pháp đo TEM. 
Kết quả trên cho thấy chỉ sau 1 quá trình acetate hóa cellulose đã đồng thời làm giảm độ phân cực và giảm đáng kể kích thước của sợi cellulose. Từ đó có thể đưa ra dự đoán về khả năng tương thích tốt của sợi cellulose đã biến tính trong nền polymer - phù hợp với định hướng làm pha gia cường trong tổng hợp vật liệu composite phân hủy sinh học.

\subsection{2. Ảnh hưởng của thời gian siêu âm đến phản ứng acetate hóa cellulose}

Các mẫu cellulose acetate sau phản ứng được rửa lại nhiều lần với nước cất để loại bỏ tác chất còn dư (anhydride acetic) và dung môi (acid acetic). Sản phẩm sau khi sấy khô được phân tích bằng phương pháp phổ IR, kết quả thể hiện trong Hình 8.
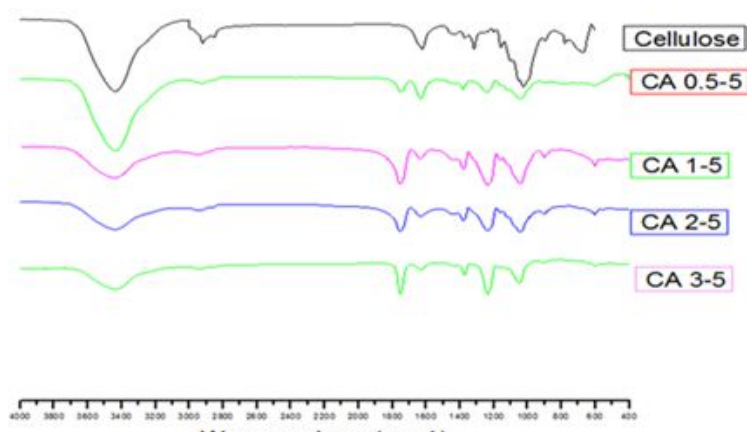

Wavenumbers $(\mathrm{cm}-1)$

Hình 8. Kết quả phổ IR các mẫu khảo sát ảnh hưởng thời gian siêu âm: Cellulose, CA $0,5-5$ ( 0,5 giờ), CA $1-5$ (1 giờ), CA $2-5$ (2 giờ), CA $3-5$ (3 giờ).

Phản ứng acetate hóa cellulose được xác nhận rõ ràng thông qua sự xuất hiện peak tại số sóng $1750 / \mathrm{cm}$ đặc trưng cho tần số dao động của liên kết $\mathrm{C}=\mathrm{O}$ của nhóm acetyl $\mathrm{CH}_{3} \mathrm{COO}-$. Kết quả cho thấy tỷ lệ cường độ peak này so với cường độ peak $3300-3500 / \mathrm{cm}$ của nhóm - OH tăng mạnh khi tăng thời gian siêu âm từ 0,5 giờ lên 1 giờ, tuy nhiên tiếp tục tăng thời gian siêu âm lên 2 giờ, 3 giờ thì tỷ lệ này không tăng thêm nữa. Điều này cho thấy sau 1 giờ, quá trình đã đạt trạng thái cân bằng, do đó thời gian siêu âm 1 giờ là phù hợp, kết quả này được chọn cho khảo sát tiếp theo.

\subsection{3. Ảnh hưởng của thể tích anhydride acetic đến phản ứng acetate hóa cellulose}

Các mẫu cellulose acetate của quá trình khảo sát ảnh hưởng của anhydride acetic được phân tích bằng phương pháp phổ IR, kết quả thể hiện trong Hình 9.

Dựa vào Hình 9 nhận thấy tăng thể tích anhydride acetic từ 5 lên $10 \mathrm{~mL}$, tỷ lệ cường độ peak tại số sóng $1750 / \mathrm{cm}$ so với peak $3300-3500 / \mathrm{cm}$ tăng mạnh, điều này chứng tỏ hiệu suất quá trình acetate hóa cellulose tăng. Tuy nhiên khi tiếp tục tăng thể tích anhydride acetic từ 10 lên $13 \mathrm{~mL}$ thì tỷ lệ này không tăng thêm nữa, điều này cho thấy $10 \mathrm{ml}$ anhydride acetic là phù hợp cho phản ứng.

Quá trình biến tính cellulose bằng anhydride acetic để acetate hóa một số nhóm -OH đã bước đầu đạt hiệu quả, điều kiện phù hợp cho quá trình này là: $0,5 \mathrm{~g}$ bột cellulose, $10 \mathrm{~mL}$ anhydride acetic trong dung môi acid acetic, xúc tác $\mathrm{H}_{2} \mathrm{SO}_{4}$ đặc và siêu âm trong 1 giờ.

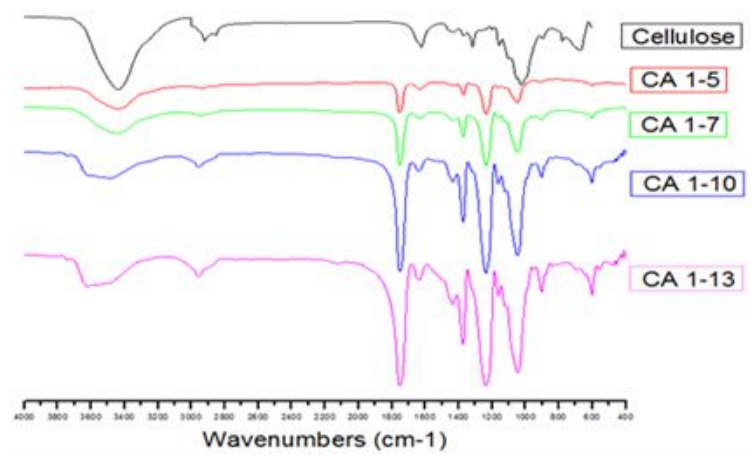

Hình 9. Kết quả phổ IR các mẫu khảo sát ảnh hưởng thể tích anhydride acetic: Cellulose, CA 1 - 5 (5 mL), CA 1 - 7 (7 mL), CA 1 - 10 (10 mL), CA 1 - 13 (13 $\mathrm{mL})$.

\section{Kết Luận}

Nghiên cứu đã rút ngắn được quy trình xử lý: có thể thu được cellulose chỉ qua một bước xử lý $\mathrm{NaOH}$, hemicelluloses và lignin trong lục bình đã được loại bỏ. Điều kiện thích hợp cho quá trình này là: $0,5 \mathrm{~g}$ lục bình khô trong $25 \mathrm{~mL}$ dung dịch $\mathrm{NaOH} 0,25 \mathrm{M}$, thời gian 5 giờ và nhiệt độ được duy trì $80^{\circ} \mathrm{C}$. Cellulose được biến tính bằng anhydride acetic nhằm làm giảm độ phân cực của cellulose, điều kiện phù hợp cho phản ứng là: $0,5 \mathrm{~g}$ bột cellulose, $10 \mathrm{~mL}$ anhydride acetic và siêu âm trong 1 giờ bằng máy siêu âm Elmasonic $\mathrm{S} 100$ $\mathrm{H}$ cường độ $500 \mathrm{~W}$. Kết quả thu được vi sợi cellulose acetate kích thước khoảng 50 nm, độ phân cực giảm mạnh so với cellulose ban đầu, phù hợp làm pha gia cường cho vật liệu nanocomposite phân hủy sinh học. 


\section{Lời Cảm Ơn}

Nghiên cứu này được hỗ trợ kinh phí bởi đề tài cơ sở Trường Đại học Nông Lâm TP.HCM. Mã số: CS-CB17-KH-04.

\section{Tài Liệu Tham Khảo (References)}

Abdulkhani, A., Hosseinzadeh, J., Ashori, A., Dadashi, S., \& Takzare, Z. (2014). Preparation and characterization of modified cellulose nanofibers reinforced polylactic acid nanocomposite. Polymer Testing 35, 73-79.

Istirokhatun, T., Rokhati, N., Rachmawaty, R., Meriyani, M., Priyanto, S., \& Susanto, H. (2015). Cellulose isolation from tropical water hyacinth for membrane preparation. Procedia Environmental Sciences 23, 274-281.
Nguyen, T. B. T., Luong, T. H. V., Tran, L. N. P., Nguyen, T. T. D., \& Yi-Hsu, J. (2017) Comparison of some pretreatment methods on cellulose recovery from water hyacinth (Eichhornia Crassipe). Journal of Clean Energy Technologies 5(4), 274-279.

Oka, M. A. (2010). PLA and cellulose based degradable polymer composites. (Doctoral dissertation). Georgia Institute of Technology, Georgia, USA.

Wang, T., \& Drzal, L. T. (2012). Cellulose-nanofiberreinforced poly (lactic acid) composites prepared by a water-based Approach. ACS Applied Materials \& Interfaces 4(10), 5079-5085. 